\title{
Magellanic Cloud novae and the peak magnitude vs rate-of-decline relationship
}

\author{
William Liller \\ Center for Nova Studies, Casilla 5022 Reñaca, Viña del Mar, Chile \\ Raquel Yumi Shida \\ Departamento de Astronomia, IAG/USP, Rua do Matão 1226, Cidade \\ Universitária, 05508-900 São Paulo SP, Brazil
}

\begin{abstract}
Studying classical novae in the Magellanic Clouds has at least three huge advantages: (1) their distances are well-known; (2) the interstellar extinction is small and reasonably well known; and (3) an abundance of novae (47 as of 2003 September 15) have been discovered in the two clouds. Consequently, we will investigate in this paper one of the most important characteristics of novae: the relationship between the absolute magnitudes of the nova outbursts at maximum brightness and their rate of decline, often referred to as the MMRD.
\end{abstract}

\section{Introduction}

Many investigators have expended considerable effort to derive the MMRD using Galactic novae, one of the most recent and careful studies being that of Downes \& Duerbeck (2000); see this paper for an excellent summary of the previous work done on this problem. They derive distances from the rates of expansions of the nova shells, but note that systematic errors can arise owing to effects such as non-spherical mass ejection or deceleration of the shell material.

The special problems that will be encountered here are several: determining precisely the value of the magnitude at maximum brightness since Magellanic Cloud novae are not, in general, as well observed as galactic novae; finding a single and reliable value for the rate of decline of the nova brightness using the scattered inhomogeneous observations, especially those of earlier investigators; and deriving a consistent set of magnitudes from observations made with a variety of passbands.

We have what we believe to be reliable values for the brightnesses and rates of decline of many of the 47 classical novae known to have occurred to date, and for a number of recently discovered novae we have been able to use unpublished data coming primarily from the nova search program of the first author. Because of the ongoing nature of this project and space restrictions imposed in this conference volume, the fully analyzed data will be published elsewhere. However, we present here preliminary MMRD diagrams. We have adopted the distance moduli and extinction values given by van den Bergh (2000), namely 18.50 and $0.40 \mathrm{mag}$, respectively, for the LMC, and 18.85 and $0.19 \mathrm{mag}$ for the SMC. The 
locations of all the novae in the Magellanic Clouds are shown in Shida \& Liller (these proceedings).

Three MMRD relations appear below with figures showing different kinds of curves representing the plotted points. The magnitudes at maximum brightness, both apparent and absolute, and the rates of decline, both in units of the usual $t_{2}$ (on a logarithmic scale) and the logarithm of 100 times the daily rate of fading, are plotted for 36 novae, with the following conventions: filled circles and plus signs represent well-determined and less-well-determined values for the LMC, respectively; filled diamonds and crosses correspond similarly to the SMC. An open square indicates data taken from unpublished results. Data from the MACHO, EROS2, OGLE and MOA surveys are shown as triangles and should be regarded as indicating "equal to or brighter". Note that in these figures the magnitudes of the SMC novae have been adjusted to the scale of the LMC.

Fig. 1 shows the individual points together with a straight line that fits the observations reasonably well, namely

$$
M_{v}=-10.00+1.39 \log t_{2} .
$$

The linear relationship derived by Downes \& Duerbeck differs considerably with the two coefficients being -11.32 and +2.55 ; our less steep line crosses the Downes \& Duerbeck line at $M_{v}=-8.42$. As can be seen, we have given the BdeV-26 point considerable weight and have taken as well determined the bulk of points in the center of the graph. (BdeV-26 represents the 1926 nova in the LMC studied by Buscombe \& de Vaucouleurs 1955).

Fig. 1 also shows the "S-shaped curve" first suggested by Capaccioli et al. (1989). In our judgement, the best representation differs little from the expression given by Downes \& Duerbeck, viz.

$$
M_{v}=-8.02-1.23 \arctan \left[\left(1.32-\log t_{2}\right) / 0.23\right] .
$$

The reason our conclusions are now similar to Downes \& Duerbeck's is that the S-shaped curve follows well the sudden fading indicated by the several slow nova points with $\log t_{2}>\sim 1.6$ and gives importance to the bulk of the points in the center of the graph. It can be seen from the figure that this curve tends to form an upper envelope to the well-observed points. Since it is nearly always impossible to know exactly the instant of the maximum of each of the nova light curves, many of these magnitudes, even when seemingly well determined, must be regarded as "equal to or brighter" values.

Finally, we show in Fig. 2 a quadratic expression, namely

$$
M_{v}=-9.60+0.28 \log t_{2}+0.70\left(\log t_{2}\right)^{2} .
$$

We find this expression interesting because it has never to our knowledge been used before to describe an MMRD. As can be seen, it does quite well representing the Magellanic Cloud observations.

\section{Discussion}

It should be noted that Subramaniam \& Anupama (2002) derived color-magnitude diagrams for stars within a few arcminutes of 15 LMC novae and found that in 


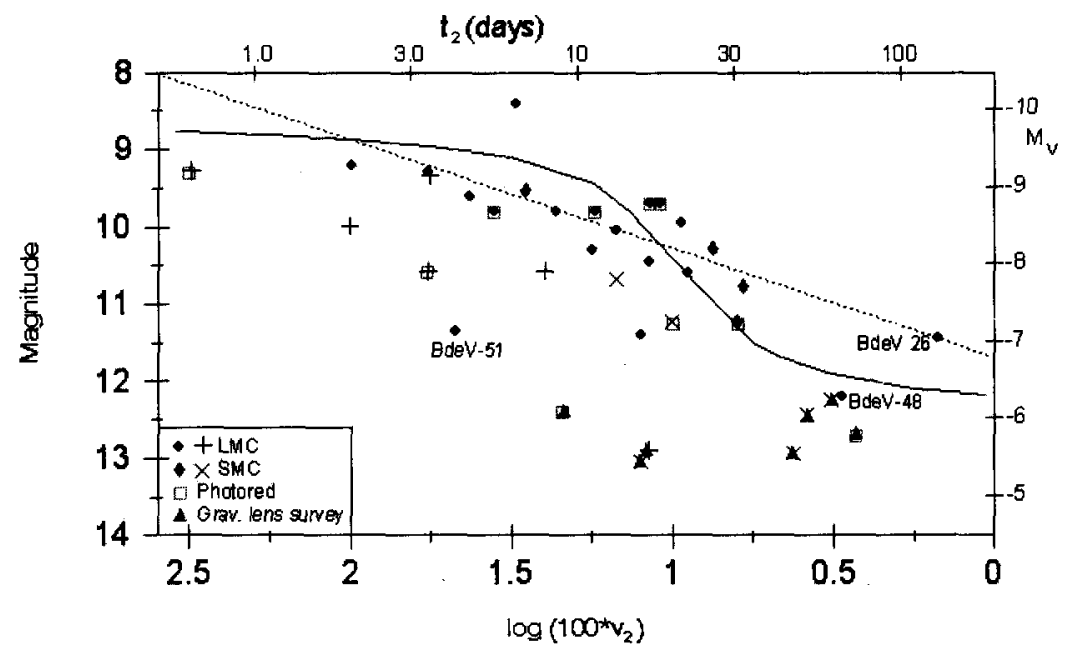

Figure 1. MMRDs for the Magellanic Clouds as represented by a straight line, and by the S-curve defined by Capaccioli et al. (1989). The meanings of the various symbols are given in the text

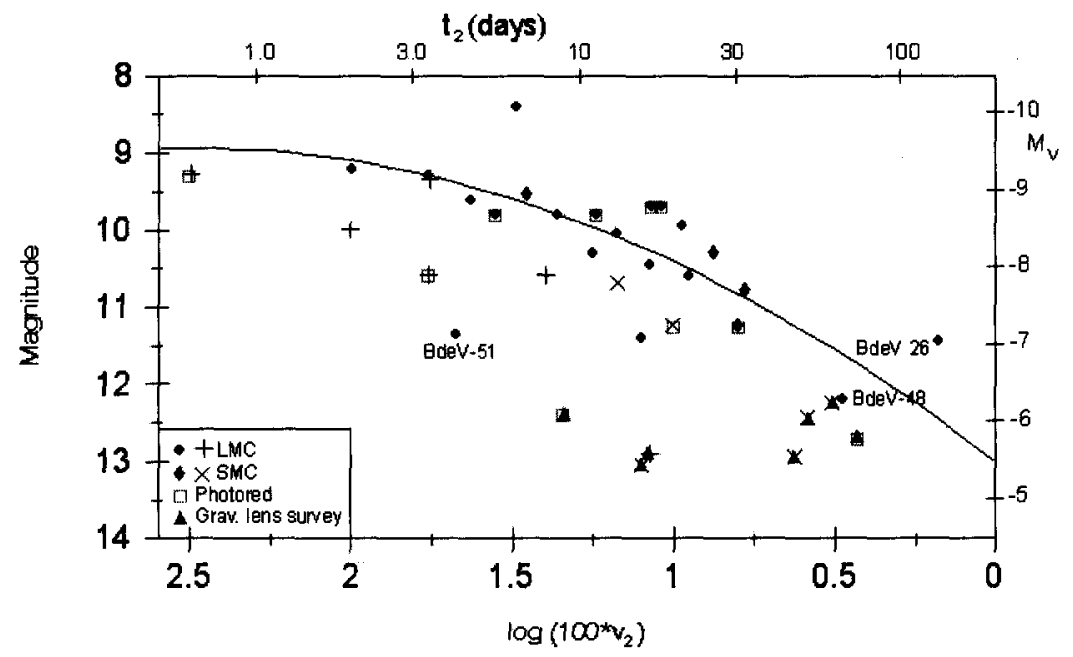

Figure 2. The MMRD for the Magellanic Clouds as represented by a quadratic curve. The meanings of the various symbols are given in the text 
only one case, Nova LMC 1948, was the assumption that a single value for reddening unsatisfactory.

In a separate paper (in preparation), we will, besides listing all the relevant data, apply appropriate weighting factors to each of the points so that the coefficients of the three expressions given above can be derived quantitatively. However, these results clearly point out the value of using Magellanic Cloud novae to determine a universal MMRD - and reveal some of the problems associated with its derivation.

\section{Acknowledgements}

We should very much like to thank Drs Pam Kilmartin and Doug Welch for their able assistance. This paper utilizes public domain data obtained by the MACHO Project, jointly funded by the US Department of Energy through the University of California, Lawrence Livermore National Laboratory under contract No. W7405-Eng-48, by the National Science Foundation through the Center for Particle Astrophysics of the University of California under cooperative agreement AST8809616 , and by the Mount Stromlo and Siding Spring Observatory, part of the Australian National University. We also thank the EROS, MOA and OGLE projects.

\section{References}

Buscombe, W., de Vaucouleurs, G. 1955, Observatory, 75, 170

Capaccioli, M., Della Valle, M., D'Onofrio, M., Rosini, L. 1989, AJ, 97, 1622

Downes, R.A., Duerbeck H.W. 2000, AJ, 120, 2007

Subramaniam, A., Anupama, G.C. 2002, A\&A, 390, 449

van den Bergh, S. 2000, in The Galaxies of the Local Group (Cambridge University Press), 143

\section{Discussion}

Feast: Can you say how complete your nova survey is likely to be?

Liller: Since 1990 the NASA and STScI nova experts have asked me to cover the Magellanic Clouds more intensively. So I would say that since then, the survey has been reasonably complete. 
534

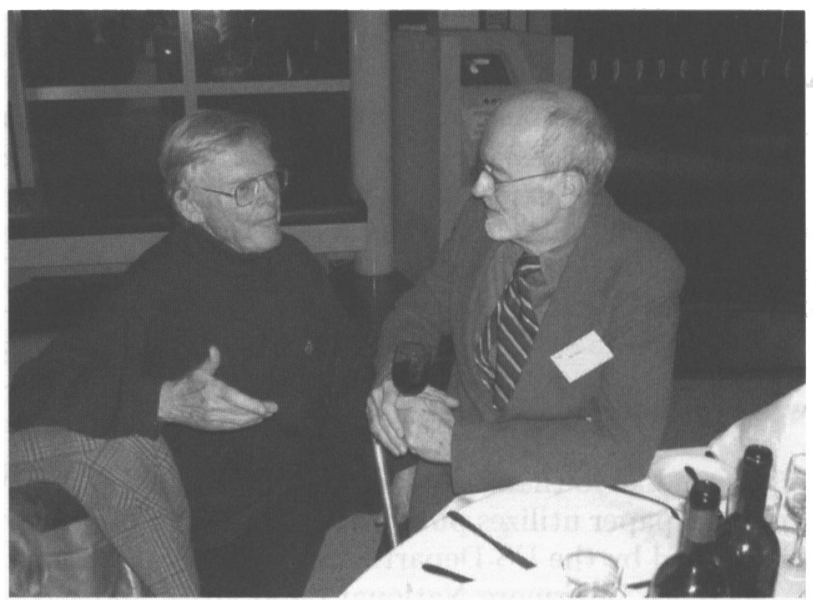

Bill Liller and Michael Snowden

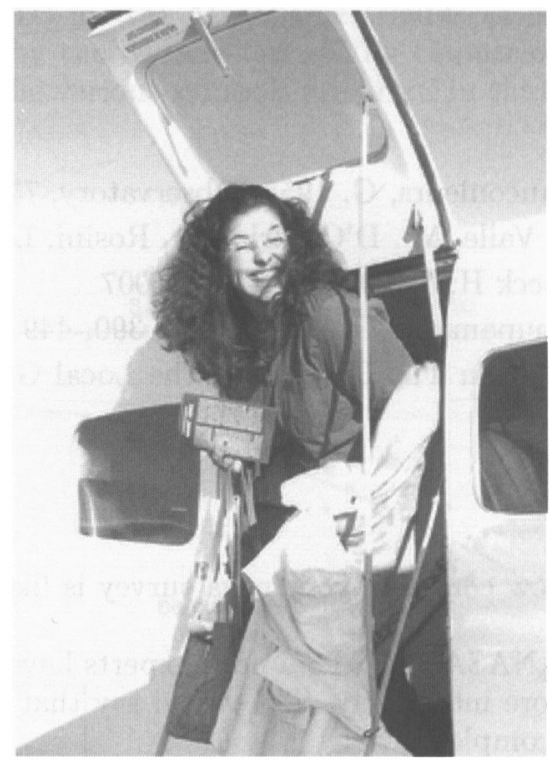

Merieme Chadid 